\section{A case of lipomembranous panniculitis with a dramatic response to the treatment of venous insufficiency}

\author{
Didem Mullaaziz, ${ }^{1}$ Aslı Kaptanoğlu, ${ }^{2}$ \\ Emel Erdal Çalıkoğlu, ${ }^{1}$ \\ Hanife Özkayalar ${ }^{3}$
}

${ }^{1}$ Department of Dermatology and Venereology, Near East University Faculty of Medicine, Nicosia, North Cyprus; '2Department of Dermatology and Venereology, Marmara University Faculty of Medicine, İstanbul, Turkey; ${ }^{3}$ Department of Pathology, Faculty of Medicine, Near East University, Nicosia, Cyprus

\begin{abstract}
Lipomembranous panniculitis (LP) is a peculiar type of fat necrosis and is reported with various clinical conditions, mostly with peripheral vascular diseases. Here, a case of a 57-year-old woman with a painful erythematous swelling of the right leg in association with venous insufficiency has been reported. Although LP is frequently associated with venous insufficiency, to the best of our knowledge, this is the first report on the beneficial effect of venous insufficiency treatment in LP.
\end{abstract}

\section{Introduction}

Lipomembranous panniculitis (LP) refers to a chronic inflammation of the subcutaneous tissue, presumably representing a non-specific type of ischemic necrosis of the fatty tissue. ${ }^{1}$ There are several underlying associated clinical disorders such as diabetes, erythema nodosum, morphea and lupus panniculitis, but the most commonly noted one is venous insufficiency. ${ }^{2}$ It is generally characterized by painful sclerotic changes, which are observed on the lower limbs of overweight, middle-aged, diabetic women with severe venous stasis. ${ }^{1}$ Histologic changes of this panniculite type may be the result of an inflammatory reaction in patients who have the liposclerosis of venous insufficiency with connective tissue disease or previous leg ischemia or both. ${ }^{3}$ Although LP is frequently associated with venous insufficiency, to the best of our knowledge, this is the first report on the beneficial effect of venous insufficiency treatment in LP.

\section{Case Report}

A 57-year-old woman known to have diabetes mellitus presented a right leg swelling for 2 years. She noted erythema and swelling of the right leg accompanied by pain. Dermatological examination revealed an erythematous, violaceous, indurated sclerotic plaque on the posterolateral aspect of the right tibia. Multiple varicose veins were located in the posterior tibial area. No history of trauma or discharge was recorded. She had a history of thrombophlebitis on the same leg and this happened twice, one of which was four years ago and the other one was six years ago. Doppler ultrasound revealed no evidence of deep vein thrombosis. Bilateral dilate varicose veins were detected, which were more prominent on the right leg (Figure 1).

The differential diagnosis consisted of erythema nodosum, erythema induratum, nodular vasculitis, alpha-1 antitrypsin deficiency-associated panniculitis, traumainduced panniculitis, morfea, necrobiosis lipoidica, cutaneous sarcoidosis and subcutaneous granuloma annulare. A punch biopsy was performed for the histopathological evaluation. The subcutaneous fat had microcystic changes within the fat lobules (Figure 2). The membrane caused small pseudopapillae with periodic acid-Schiff stain (Figure 3). Macrophage accumulation was found in the subcutaneous tissue (Figure 4). Fibrozis was present in the reticular dermis (Figure 5). These histological findings allowed the diagnosis of the LP.

Complete blood count, liver and kidney function tests, electrolytes, erythrocyte sedimentation rate, $\mathrm{C}$ reactive protein, thyroid function tests, serum complement levels, alpha-1 antitrypsin, serum amylase and lipase levels were all observed to be within the normal limits. Antinuclear antibody, rheumatoid factor, thyroid autoantibodies were found to be negative. The patient was observed to be hyperglycemic, with a blood glucose level of 127 . Other test results were observed to be within the normal limits. Systemic steroid treatment was started with a daily dosage of $60 \mathrm{mg}$. The treatment was completed in 3 months by reducing it $10 \mathrm{mg}$ within the intervals of 2 weeks. Relapse was observed 1 week after the steroid therapy and the colchicine therapy was started. Partial regression was observed in the existing lesions during the 2-month follow-up period with colchicine treatment, but the formation of new lesions continued and the treatment was terminated on her own request. For the venous insuffiency, a consultation of cardiovascular surgery was performed and right saphena
Correspondence: Didem Mullaaziz, Department of Dermatology and Venereology, Near East University Faculty of Medicine, Near East Boulevard, 99138 Nicosia, North Cyprus Mersin 10, Turkey.

Tel.: +903926751000/1277.

E-mail: mullaazizdidem@gmail.com; didem mullaaziz@yahoo.com

Key words: Lipomembranous panniculitis; panniculitis; fat necrosis; venous insufficiency.

Contributions: the authors contributed equally.

Conflict of interest: the authors declare no potential conflict of interest.

Received for publication: 7 November 2017. Accepted for publication: 30 April 2018.

This work is licensed under a Creative Commons Attribution-NonCommercial 4.0 International License (CC BY-NC 4.0).

CCopyright D. Mullaaziz et al., 2018

Licensee PAGEPress, Italy

Dermatology Reports 2018; 10:7486

doi:10.4081/dr.2018.7486

manga was treated by the radiofrequency ablation method in addition to the calcium dobesilate medical treatment, No new lesions occured during the 2 year follow-up period after the surgical treatment (Figure 6).

\section{Discussion}

LP was first described in the NasuHakola disease, which is characterized by the membranocystic degeneration of long bones and adipose tissues as well as the sudanophilic leukodystrophy of cerebral hemispheres. However, subsequent reports demonstrated that the membranocytic changes could be associated with arterial ischemia and venous insufficiency. ${ }^{4}$

In a series of 38 cases with LP, the mean age of all patients was $57,89 \%$ of them were women and the majority were obese, similar to our case. LP can demonstrate very different clinical manifestations such as sclerotic plaque, subcutaneous nodule, atrophic plaque, ulceration and purpura. The most common clinical context was chronic sclerotic plaque and it was most commonly observed on the lower leg $(82 \%)$. In our case, a sclerotic plaque was observed on the lower limb. Other reported locations included the buttock, the trunk, and the arm. There are several reports of associated clinical disorders such as venous 


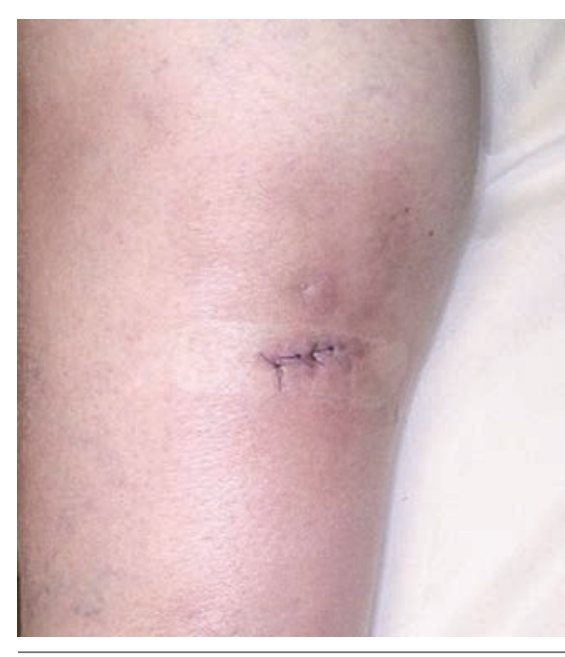

Figure 1. Erythematous, indurated sclerotic plaque on the posterolateral aspect of the right tibia.

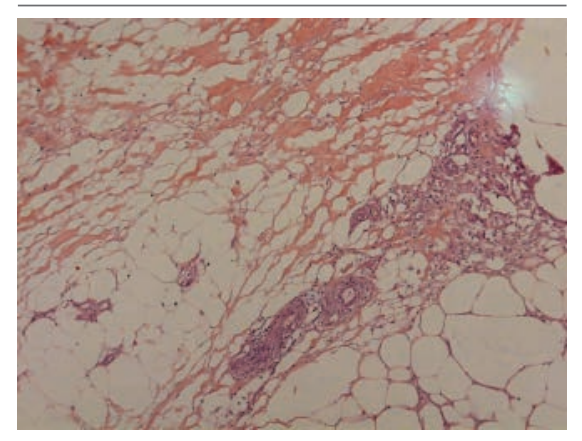

Figure 2. The subcutaneous fat had microcystic changes within the fat.

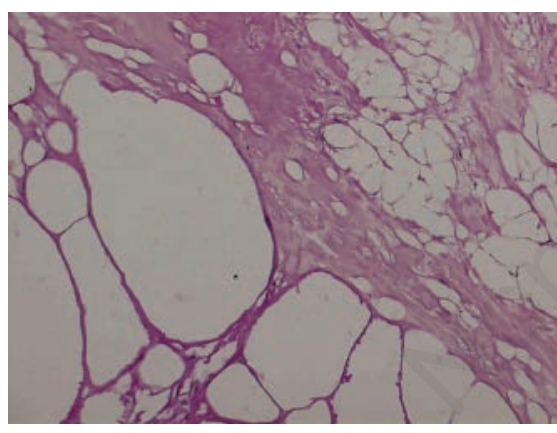

Figure 3. The membrane formed small pseudopapillae (PAS x20).

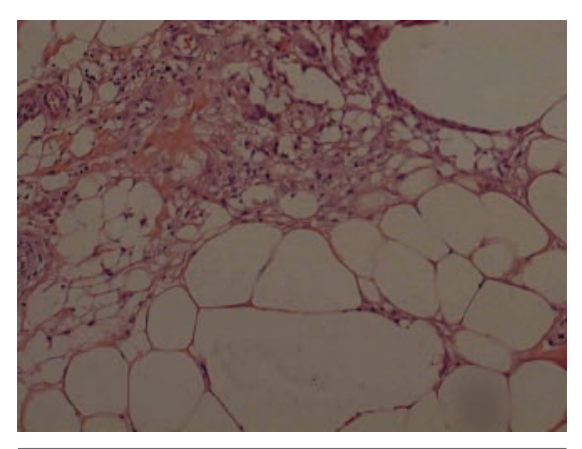

Figure 4. Macrophage accumulation was found inthe subcutaneous tissue (HE X20). insufficiency, stasis dermatitis, hemosiderin pigmentation, overt venous varicosities, erythema nodosum, morphea, and lupus panniculitis but the venous insufficiency is the most common association. It has been concluded that the lipomembranous fat necrosis is most likely to be a nonspecific form of the ischemic fat degeneration that may be induced by various clinical entities. This change is most often seen in venous insufficiency-associated chronic sclerotic plaques, typically observed in middle-aged obese women, and the term stasis-associated lipomembranous panniculitis (SALP) is proposed in order to describe this most common form of lipomembranous fat necrosis. ${ }^{5}$

In addition to venous insufficiency psoriasis, multiple colon polyposis, ulcerative colitis, celiac disease, ${ }^{3}$ rheumatoid arthritis, ${ }^{4}$ morphea, ${ }^{6}$ dermatomyositis, ${ }^{7}$ liver cirrhosis and diabetes ${ }^{2}$ have been reported to be associated with the LP. Otherwise, one case has been reported to be associated with trauma ${ }^{8}$ and two cases were reported to be associated with chemotherapy. ${ }^{8-10}$

In regard of the occurrence period of the lesions, it is reported that most of the lesions had been evolving for over 6 months and they were associated with chronic venous insufficiency of the legs in a series

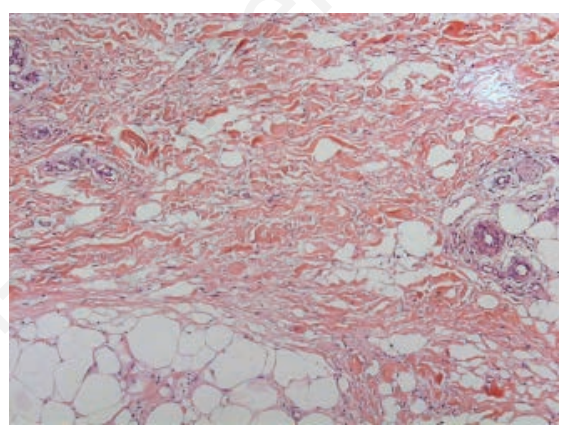

Figure 5. Fibrozis was found in reticular dermis (HE X 10).

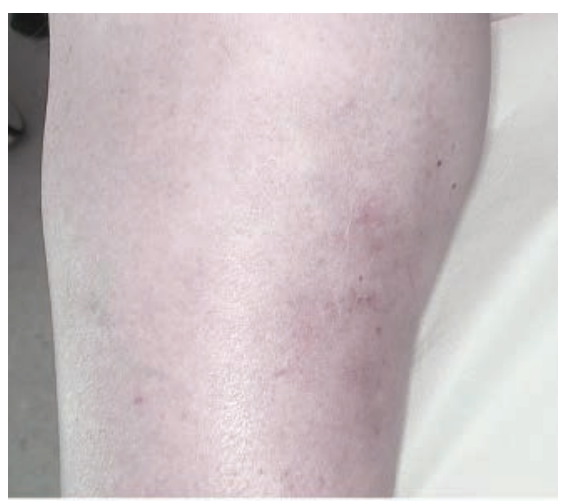

Figure 6. After 6 months of treatment. of 8 cases with LP. In two of the cases, the lesions occurred in patients with connective tissue disorders. ${ }^{1}$ In our case, the lesions were chronic and they were present for 2 years. There was no associated connective tissue disease.

The histological features of LP are characterized by thickened undulating membrane lining microcysts. Projection of the membranes into the cystic spaces is apparent. ${ }^{4}$ In one series, it was associated with other variable histological features including dilated veins, hemorrhage, endarteritis obliterans, and sclerosis. Few cases also demonstrated capillaries proliferation, calcified vessels, and hemosiderin deposition. ${ }^{3}$

LP is predominantly associated with venous insufficiency, arterial ischemia, and the presence of varicosity. Moreover, the histological findings revealed vascular pathologies. These findings suggest that LP is caused by fatty tissue ischemic damage. Ischemic damage leads to the degeneration of fat tissues and fibrinogen accumulation leading to the characteristic histology of LP. ${ }^{3}$ Other hypotheses explained these changes to be caused by the circulatory disturbances and the metabolic disorder of lipids. ${ }^{5}$

The treatment mostly depends on the relief of associated conditions targeting the inhibition of inflammation and fibrosis. Recently there is no standart therapy of this condition. In our case, conventional treatments were not successful whereas a surgical approach for venous insufficiency was successful for the relief of the symptoms.

\section{Conclusions}

In conclusion, in case of a presence of sclerotic plaque in the lower extremity in a female patient with middle age and obesity, venous insufficiency and LP should be considered in the differential diagnosis. Also, in patients of LP with venous insuffficiency, relief of vascular problems with surgery may be an alternative option when resistant to conventional anti-inflamatorry therapy. Therefore, increased number of cases may enlighten the way for pathogenesis, its associations and alternative treatment options for the resistant cases.

\section{References}

1. Gouveia C, Soares Almeida LM. [Lipomembranous panniculitis: clinicopathologic correlation of 8 cases]. [Article in Spanish]. Actas Dermosifiliogr 2006;97:379-84.

2. Al-Brahim N, Ceballos K, Sur M, et al. 
Lipomembranous panniculitis: report of a case. Ann Diagn Pathol 2007;11:2824.

3. Alegre VA, Winkelmann K, Aliaga A. Lipomembranous changes in chronic panniculitis. J Am Acad Dermatol 1988;19:39-46.

4. Asadi-Kani Z, Moravvej H, Gaisari M, Kavand S, Saeedi M. A Case of Lipomembranous Panniculitis Associated with Rheumatoid Arthritis. Iran J Dermatol 2009;12:27-9.

5. Snow JL, Su WP. Lipomembranous (membranocystic) fat necrosis Clinicopathologic correlation of 38 cases. Am J Dermatopathol 1996;18:151-5.

6. Aounallah A, Zaraa I, El Euch D, et al. Lipomembranous panniculitis associated with venous insufficiency, morphea, and psoriasis. Skinmed 2016;14:127-9.

7. Ishikawa O, Tamura A, Ryuzaki K, et al. Membranocystic changes in the panniculitis of dermatomyositis. $\mathrm{Br} \mathrm{J}$ Dermatol 1996;134:773-6.

8. Wong JJ, Greenberg RD. Upper extrem- ity nodules. Arch Dermatol 2004;140:231-6.

9. Pincemaille B, Besançon C, Balme B, et al. Membranous lipodystrophy caused by chemotherapy. Ann Dermatol Venereol 1998;125:425-8.

10. Akay OM, Urer SM, Oner U, Gulbas Z. Lipomembranous panniculitis in a patient with acute leukemia induced by chemotherapy. Leuk Res 2008;32:66971. 